\title{
Empirical Mode Decomposition of the atmospheric wave field
}

\author{
A. J. McDonald, A. J. G. Baumgaertner, G. J. Fraser, S. E. George, and S. Marsh \\ Department of Physics and Astronomy, University of Canterbury, Christchurch, New Zealand
}

Received: 6 July 2006 - Revised: 4 January 2007 - Accepted: 15 January 2007 - Published: 8 March 2007

\begin{abstract}
This study examines the utility of the Empirical Mode Decomposition (EMD) time-series analysis technique to separate the horizontal wind field observed by the Scott Base MF radar $\left(78^{\circ} \mathrm{S}, 167^{\circ} \mathrm{E}\right)$ into its constituent parts made up of the mean wind, gravity waves, tides, planetary waves and instrumental noise. Analysis suggests that EMD effectively separates the wind field into a set of Intrinsic Mode Functions (IMFs) which can be related to atmospheric waves with different temporal scales. The Intrinsic Mode Functions resultant from application of the EMD technique to Monte-Carlo simulations of white- and red-noise processes are compared to those obtained from the measurements and are shown to be significantly different statistically. Thus, application of the EMD technique to the MF radar horizontal wind data can be used to prove that this data contains information on internal gravity waves, tides and planetary wave motions.
\end{abstract}

Examination also suggests that the EMD technique has the ability to highlight amplitude and frequency modulations in these signals. Closer examination of one of these regions of amplitude modulation associated with dominant periods close to $12 \mathrm{~h}$ is suggested to be related to a wave-wave interaction between the semi-diurnal tide and a planetary wave. Application of the Hilbert transform to the IMFs forms a Hilbert-Huang spectrum which provides a way of viewing the data in a similar manner to the analysis from a continuous wavelet transform. However, the fact that the basis function of EMD is data-driven and does not need to be selected a priori is a major advantage. In addition, the skeleton diagrams, produced from the results of the Hilbert-Huang spectrum, provide a method of presentation which allows quantitative information on the instantaneous period and amplitude squared to be displayed as a function of time. Thus, it provides a novel way to view frequency and amplitude-

Correspondence to: A. J. McDonald

(adrian.mcdonald@canterbury.ac.nz) modulated wave phenomena and potentially non-linear interactions. It also has the significant advantage that the results obtained are more quantitative than those resultant from the continuous wavelet transform.

Keywords. Meteorology and atmospheric dynamics (Middle atmosphere dynamics; Waves and tides; Instruments and techniques)

\section{Introduction}

Empirical Mode Decomposition (EMD) is a signal processing technique developed to allow non-stationary and nonlinear time-series to be examined (Huang et al., 1998). It has already been proven to be remarkably effective in several areas of geophysical research (see articles in Huang and Shen, 2005). The central aim of this study is to examine the utility of the EMD technique to separate the observations of the horizontal wind field made by the Scott Base MF radar into the various components associated with the mean wind, gravity waves, tides, planetary waves and instrumental noise. A secondary aim of this study is to show the potential utility of the EMD technique and the Hilbert-Huang spectrum in identifying regions of non-linear interactions.

The EMD technique has previously been used by Zhu et al. (1997) to examine vertically propagating internal gravity waves measured by falling sphere observations in the middle atmosphere. Their work highlighted the connection between the fundamental intrinsic mode functions (IMFs) derived from the EMD method and WKB solutions of a dispersivedissipative wave equation. This relationship was then exploited to show that the EMD method can provide useful insights into physical processes in the middle atmosphere where dispersive-dissipative wave phenomena are dominant.

$\mathrm{Wu}$ and Huang $(2004,2005)$ and Coughlin and Tung (2004) emphasized another useful application of the EMD

Published by Copernicus GmbH on behalf of the European Geosciences Union. 


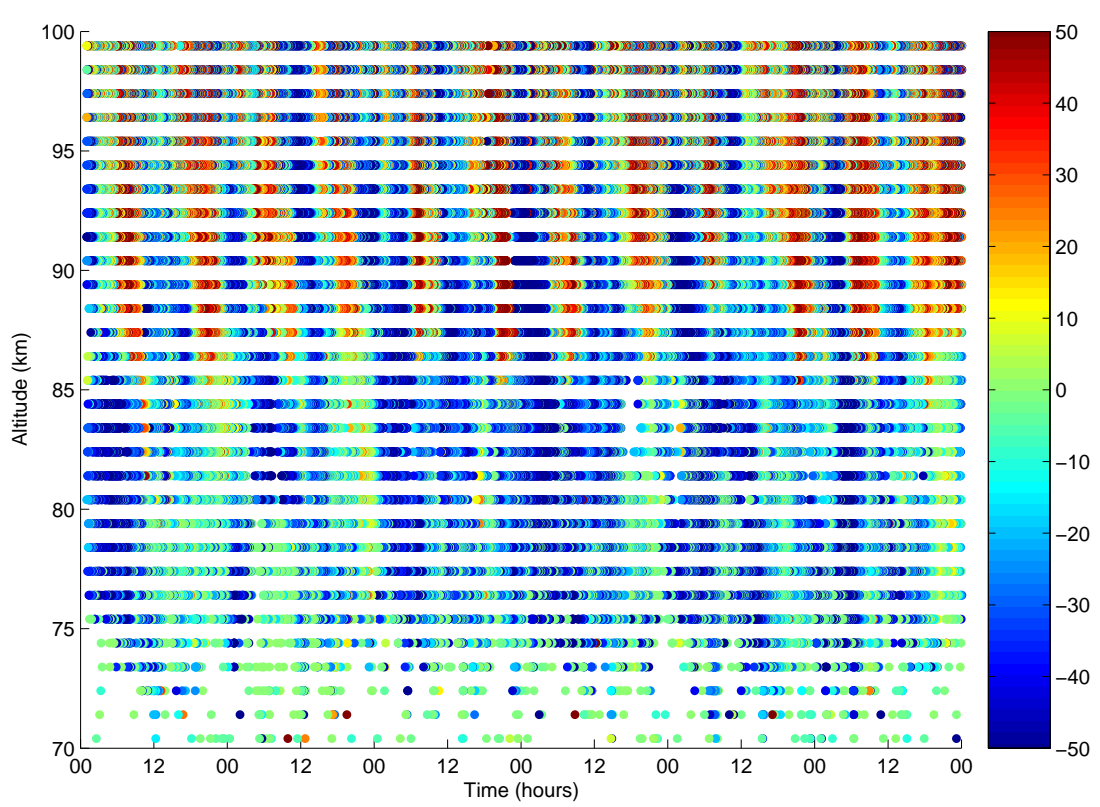

Fig. 1. Time-altitude contour plot of the zonal wind $\left(\mathrm{ms}^{-1}\right)$ observed by the Scott Base MF radar during the 1 to 6 January 2006 .

representation, namely that the technique can be used to identify natural variations in the atmosphere without the need for a priori information on the form of these variations. These studies compare the amplitudes of the IMFs resulting from data against those formed from a decomposition of pure noise to differentiate between the two. Coughlin and Tung (2004) use this technique to show that atmospheric temperatures and geopotential heights taken from the NCEP/NCAR reanalyses (Kalnay et al., 1996) can be represented by five global oscillations and a trend. Examination of the fourth mode suggests it is synchronized with the 11 -year F10.7 cm solar flux almost everywhere in the lower atmosphere. Their statistical test is used to show that this signal is different from red-noise (red-noise is defined by autocorrelated noise and has a Fourier spectrum with increasing power for decreasing frequency), indicating that there is enhanced warming in the troposphere during times of increased solar radiation. We use this technique to examine the ability of the Scott Base MF radar to observe the atmospheric wave field.

One of the most intriguing features of tides in the mesosphere-lower-thermosphere (MLT) region is their great variability. Observations have revealed fluctuations in tidal properties on time scales ranging from days to years. In particular, the winter high-latitude semidiurnal tide is very variable (Baumgaertner et al., 2005, 2006). The main factors that produce tidal variability at high latitudes are similar to those in midlatitudes and have been summarized concisely in Riggin et al. (2003). Work by Baumgaertner et al. (2005) and others has shown that tidal variability at the interannual time-scale can be linked to the solar cycle. However, the cause of modulations in tidal amplitude at day-to-day time-scales are still poorly understood. The potential of the
EMD technique and the Hilbert-Huang spectrum to examine planetary-wave modulation of the semi-diurnal tide is examined.

Janosi and Muller (2005) tested the EMD technique on daily total ozone column data from Mauna Loa. Their study indicates that the IMFs amplitude modulation is very sensitive to local changes such as random data removal or smoothing. Tests on synthetic data in Janosi and Muller (2005) highlight some of the limitations of decomposing quasi-periodic signals from noise with EMD. Nevertheless their study indicated that with careful application the EMD algorithm is helpful in identifying the dominant frequencies in the time series. This ability allows the fluctuations to be separated from the remaining background very effectively.

The current study first describes the MF radar observations and then provides a very brief summary of the EMD algorithm and the method used to form a Hilbert-Huang spectrum in Sect. 2. Section 2 also details methods utilized to improve the performance of the EMD algorithm. In Sect. 3 the utility of the EMD technique in separating the spectrum of waves observed in the mesosphere in zonal wind observations made by the Scott Base $\mathrm{MF}$ radar $\left(78^{\circ} \mathrm{S}, 167^{\circ} \mathrm{E}\right)$ is demonstrated. The observed IMFs are shown to correspond to physically meaningful wave modes. The different IMFs obtained are shown to be statistically different from whiteand red-noise using the methods described in Wu and Huang (2004, 2005) and Coughlin and Tung (2004), respectively. Specific periods of IMFs and the relationships observed between different waves are displayed. These are interpreted as non-linear interactions between planetary waves and tides and are described with reference to the theory discussed in Teitelbaum and Vial (1991). The utility of the Hilbert-Huang 
spectrum in the examination of different wave-wave interactions is shown.

\section{Methodology and observations}

The MF radar at Scott Base is a partial reflection wind radar that operates at $2.9 \mathrm{MHz}$ using a pulse width of $20 \mu \mathrm{s}$. The transmitter has a peak output power of $60 \mathrm{~kW}$ and the pulse repetition rate has recently been increased to $20 \mathrm{~Hz}$. The data acquisition has also recently been upgraded from an 8-bit to a 12-bit system which has improved the resolution and thereby the dynamic range of the measurements such that horizontal velocity data can generally be obtained from altitudes between 70 and $100 \mathrm{~km}$. The returns are oversampled to provide measurements at $1 \mathrm{~km}$ altitude spacing. Zonal and meridional winds at each altitude are then calculated by studying the motion of diffraction patterns on a number of antennae using the Full Correlation Analysis (FCA) technique at a two-minute time resolution. Further details about the system are indicated in Baumgaertner et al. (2005). The data utilized in this study consists of the time-series of zonal winds observed in January 2006. Figure 1 displays a timealtitude plot of the zonal wind observed between the 1 and 6 January 2006. This figure provides a reasonably representative view of the coverage and quality of the zonal winds observed by the Scott Base system at this time of year. Examination of Fig. 1 displays clear inclined stripes in the zonal velocity, these features correspond to wave perturbations in the observed wind field. The period of these features indicates that they are associated with atmospheric tides and the dominance of these features clearly illustrates the wavedriven nature of the circulation in the Mesosphere - Lower Thermosphere region.

The EMD method can not be defined analytically, but can effectively be described by a recursive "sifting" algorithm (Huang et al., 1998; Rilling et al., 2003). This adaptive process represents signals as sums of amplitudeand frequency-modulated components called intrinsic mode functions (IMFs). Given a time series $x_{n}$, with equal spacing $\Delta \mathrm{t}$ and $\mathrm{n}=0 \ldots \mathrm{N}-1$, the effective algorithm of EMD can be summarized as follows:

1. identify all extrema of $x_{n}$.

2. interpolate between minima (maxima), ending up with some envelope $\operatorname{emin}_{n}\left(\operatorname{emax}_{n}\right)$.

3. compute the mean of the two envelopes $\mathrm{m}_{n}=\left(\operatorname{emin}_{n}+\operatorname{emax}_{n}\right) / 2$.

4. extract the detail $\mathrm{d}_{n}=\mathrm{x}_{n}-\mathrm{m}_{n}$.

5. if $\mathrm{d}_{n}$ is an IMF, stop. Otherwise, iterate on $\mathrm{d}_{n}$ through steps 1 to 4 .
IMF convergence is achieved when the mean of $\mathrm{d}_{n}$ satisfies a pre-defined near-zero stopping criterion. Each IMF is subtracted from the data series, and the process is repeated until all the variability in the data series is decomposed into a number of these IMFs. It should be noted that even for data with a zero mean, the final residual can still be different from zero. This study utilizes the matlab implementation of the EMD technique defined in Rilling et al. (2003) which is available at http://perso.ens-lyon.fr/patrick.flandrin/emd.html. Adding all the IMFs together with the residual slow trend reconstructs the original signal without information loss or distortion. In most cases only 8 to 10 IMFs are required and therefore the method is more efficient than the traditional Fourier or wavelet decompositions which generally need a far larger number of modes to represent a comparably complicated signal (Zhu et al., 1997). In addition because the basis is adaptive (data-driven) the modes are empirically determined and not artificially constrained to have fixed amplitudes or frequencies. The basis can therefore offer a physically meaningful representation of the underlying processes, though several recent studies have shown that careful application of the technique is necessary (Huang et al., 2003; Coughlin and Tung, 2004; Janosi and Muller, 2005). These previous studies indicate that the uncertainty on individual IMFs should be examined because outliers and regions of missing data can have a significant affect on the algorithms performance. In addition, the index of orthogonality, defined in Huang et al. (1998), generally needs to be used to identify mode-mixing. Mode mixing occurs when an IMF consists of oscillations of dramatically disparate scales, mostly caused by intermittency of the driving mechanisms. When mode mixing occurs, an IMF can cease to have physical meaning by itself, suggesting falsely that there may be different physical processes represented in a mode. It should also be noted that because of the adaptive nature of the basis, there is no need for harmonics; therefore, EMD is ideally suited for analyzing data which include non-stationary and non-linear processes.

The time-series to which the EMD technique is applied in Sect. 3 represents data taken from $90 \mathrm{~km}$, this altitude being selected because the amount of poor data, based on a set of rejection criteria, is a minimum at this altitude for this period. It should be noted that it became clear during initial testing of the EMD method that outliers had a particularly deleterious effect on the results produced and therefore effective quality control of the data is essential. Therefore, to remove outliers each 2 min value at a particular altitude was compared to the mean and standard deviation of all the measurements within a three hour window. Any points more than three standard deviations from the mean were removed and replaced with a new value produced by linear interpolation. To further improve the data quality, in order that no data gaps occur in the time-series, only requires the processed 2 min resolution zonal wind data to be averaged to $15 \mathrm{~min}$ resolution. These measures mitigate the problems associated with the sensitivity of the technique to small variations of the signal 
identified by Janosi and Muller (2005) when using the EMD technique. It should be noted that averaging sometimes acts to enhance mode-mixing, but that the ensemble technique described later in this section ensures that this is not an issue in this study.

Huang et al. (2003) indicate that different sets of IMFs can be produced by changes to the sifting processes stopping criterion or alternatively by changing the time series in some other way (Janosi and Muller, 2005). This sensitivity is associated with the lack of a priori assumptions about the form of the basis function and allows us to obtain a measure of the uncertainty associated with individual IMFs. In this study a small amount of white noise, $1 \%$ of the standard deviation of the time series, is added to the original time series to produce 500 realizations. The IMFs from these realizations which meet a criteria based on the index of orthogonality (see Huang et al., 1998, for a definition) are then used to form an ensemble. Realizations that have a high index of orthogonality have a higher likelihood of mode-mixing. Thus, only using realizations that have an index of orthogonality below some pre-defined threshold significantly reduces the likelihood of mode-mixing occurring. The ensemble mean and standard deviation can then be used to identify the reliability of different features in the individual IMFs. It should be noted that the magnitude of white noise added did not significantly vary the size of the standard deviations on longer period IMFs as long as the value was small compared to the standard deviation of the data. In particular, sets of MonteCarlo simulations show that adding white-noise with standard deviations between 1 and 5\% of the standard deviation of the original time series did not affect IMFs other than the first significantly (see Sect. 3 and Fig. 5). However, adding white-noise with standard deviations above $5 \%$ began to produce additional short period modes (nearly totally associated with white-noise). It should be noted that one of the reviewers of this work introduced us to the study of $\mathrm{Wu}$ and Huang (2007) ${ }^{1}$ which details the Ensemble Empirical Mode Decomposition (EEMD) technique. The EEMD technique is a very similar method to that detailed above and is utilized to improve the performance of the EMD technique and reduce mode-mixing.

A key advantage with the EMD technique is that the IMFs produced can be transformed from temporal-space data to time-frequency space by applying a Hilbert transform to each IMF component. The Hilbert transform, $y(t)$, of any realvalued function $x(t)$ can be determined using:

$y(t)=\mathcal{H}[x(t)]=\frac{1}{\pi} P V \int_{-\infty}^{\infty} \frac{x(\tau)}{t-\tau} d \tau$

\footnotetext{
${ }^{1} \mathrm{Wu}, \mathrm{Z}$. and Huang, N. E.: Ensemble Empirical Mode Decomposition: A Noise Assisted Data Analysis Method, in preparation, 2007.
}

where PV is the principal value of the singular integral (or the Cauchy principal value) and using this definition $x(t)$ and $y(t)$ form the complex conjugate pair. With the Hilbert transform, the analytic signal is defined by:

$z(t)=x(t)+i y(t)=a(t) e^{i \theta(t)}$

where

$a(t)=\sqrt{x^{2}+y^{2}}$

and

$\theta(t)=a \tan \left(\frac{y}{x}\right)$

where $a(t)$ in Eq. (3) is the instantaneous amplitude and $\theta(t)$ in Eq. (4) is the instantaneous phase. The instantaneous frequency is then simply defined as the time derivative of the instantaneous phase. The amplitude and instantaneous frequency for every IMF at every time-step can thus be computed. This result can be projected on the timefrequency-energy space, with energy defined as the amplitude squared. The resulting timefrequency-energy representation of the data is called the Hilbert-Huang spectrum and provides information similar to that identified in a continuous wavelet transform (Torrence and Compo, 1998). This process and the utility of the Hilbert-Huang spectrum is discussed in detail in Huang et al. (1998) and Huang and Shen (2005).

\section{Results}

The result of application of the EMD technique to the zonal wind time series introduced in Sect. 2 is displayed in Fig. 2. The upper panel in this figure displays the original time series. The second panel contains the summation of the ensemble means of the first to third IMFs, the third panel contains the ensemble mean of the fourth IMF, the fourth panel the ensemble mean of the fifth IMF etc. Note EMD decomposes this month long time-series at 15 min resolution into 8 separate intrinsic mode functions and a residual which represents the trend over the month. The first to third IMFs have been combined in Fig. 2 because these modes all have dominant period below $8 \mathrm{~h}$ and are therefore likely to be associated with internal gravity waves. Examination of the individual IMFs used in the summation also suggests that they are likely to be related to a mixture of instrumental noise and wave-like motions. The dominant period of the fourth and fifth IMFs are associated with periods near 12 and $24 \mathrm{~h}$ and are therefore related to the semi-diurnal and diurnal tides, respectively. The sixth IMF and higher are related to periods above 2 days and are related to planetary waves. It should be noted that these wave modes, semi-diurnal and diurnal tides and planetary waves, are frequently observed in the Scott Base MF radar data (Baumgaertner et al., 2005, 2006). Thus, the fact that the EMD technique decomposes the zonal wind 


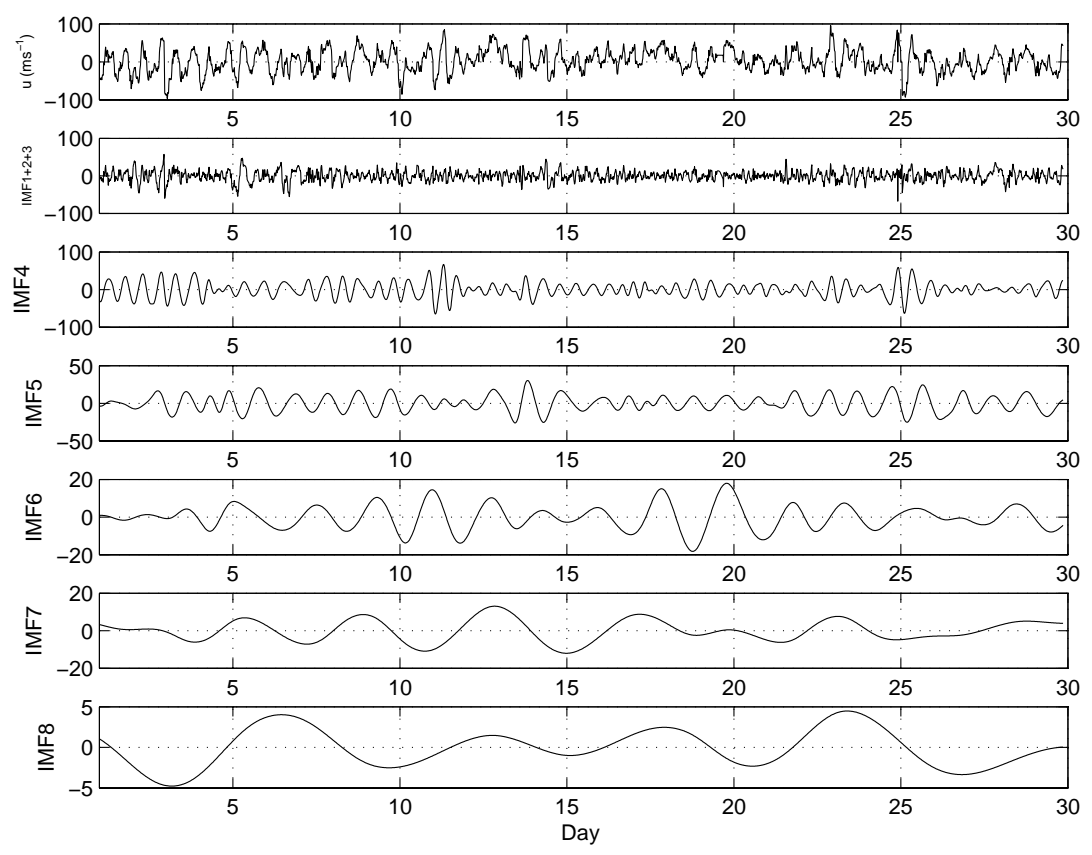

Fig. 2. The results of application of the EMD technique to the zonal wind time series observed by the Scott Base radar at $90 \mathrm{~km}$ in January 2006 are displayed. The upper panel in this figure displays the original time series. The second panel contains the summation of the first to third IMFs, the third panel contains the fourth IMF, the fourth panel the fifth IMF etc.

time series into a set of physically meaningful modes without any necessity to define a basis function gives us confidence that the technique is of significant utility. This fact also suggests that the residual calculated is an accurate representation of any trend and therefore potentially of utility in examining the mean wind. It should be noted that the Empirical Mode Decomposition technique produces results which are qualitatively similar to the output from wavelet multiple resolution analysis as used in previous studies, such as Malinga and Poole (2002a,b). However, several studies have indicated that the need to select an appropriate "mother wavelet" when using wavelet analysis limits the applicability of the technique (Torrence and Compo, 1998). It is interesting that many of the higher IMFs, principally those associated with tidal and planetary wave periods, are characterized by bursts of wave activity and thus the fact that the technique is useful in the examination of non-stationary data is valuable in this case. Thus, suggesting that application of the EMD technique may be of utility in understanding the variability of these waves. Previous work by Pancheva et al. (2002) has indicated that planetary-wave activity often occurs as "bursts" of wave activity at a particular period.

To examine the utility of the MF radar data to observe these different wave motions, a technique discussed in $\mathrm{Wu}$ and Huang $(2004,2005)$ is utilized. Their study detailed methods which allow the IMFs obtained from data to be differentiated from those which result from white-noise processes which are uniformly and normally distributed, respec- tively. Figure 3 displays a log-log diagram of the normalized versions of the energy and dominant period of the different intrinsic modes identified by the EMD technique. The full lines in Fig. 3 represent the range of values within which 75 and $99 \%$ of the simulations produced using purely whitenoise would occur, these lines being calculated from the theory indicated in Wu and Huang $(2004,2005)$. The red and blue dots indicate the result of one hundred Monte-Carlo simulations of white-noise. The green crosses in Fig. 3 represent the value of the energy and the dominant period of IMFs which result from application to the MF radar time series. To ensure the compatibility of the measurements and the MonteCarlo simulations the time-series are normalized. Examination of Fig. 3 indicates that the IMFs produced by application of the EMD technique to the MF radar zonal wind data are significantly different from the simulated and theoretical expectations for a white-noise process. It should be noted that the highest IMFs have not been included in this diagram for clarity because of the large inaccuracies in identifying long dominant periods. The separation between the IMFs associated with the observations and Monte-Carlo simulations of white-noise displayed in Fig. 3 indicates that all the IMFs (which have previously been related to atmospheric waves) are statistically different from white-noise. It is interesting to note that for randomly generated time-series, the EMD method exhibits a period doubling phenomenon so that each mode tends to have an average period of about twice the previous mode. This phenomenon creates the clustering of the 


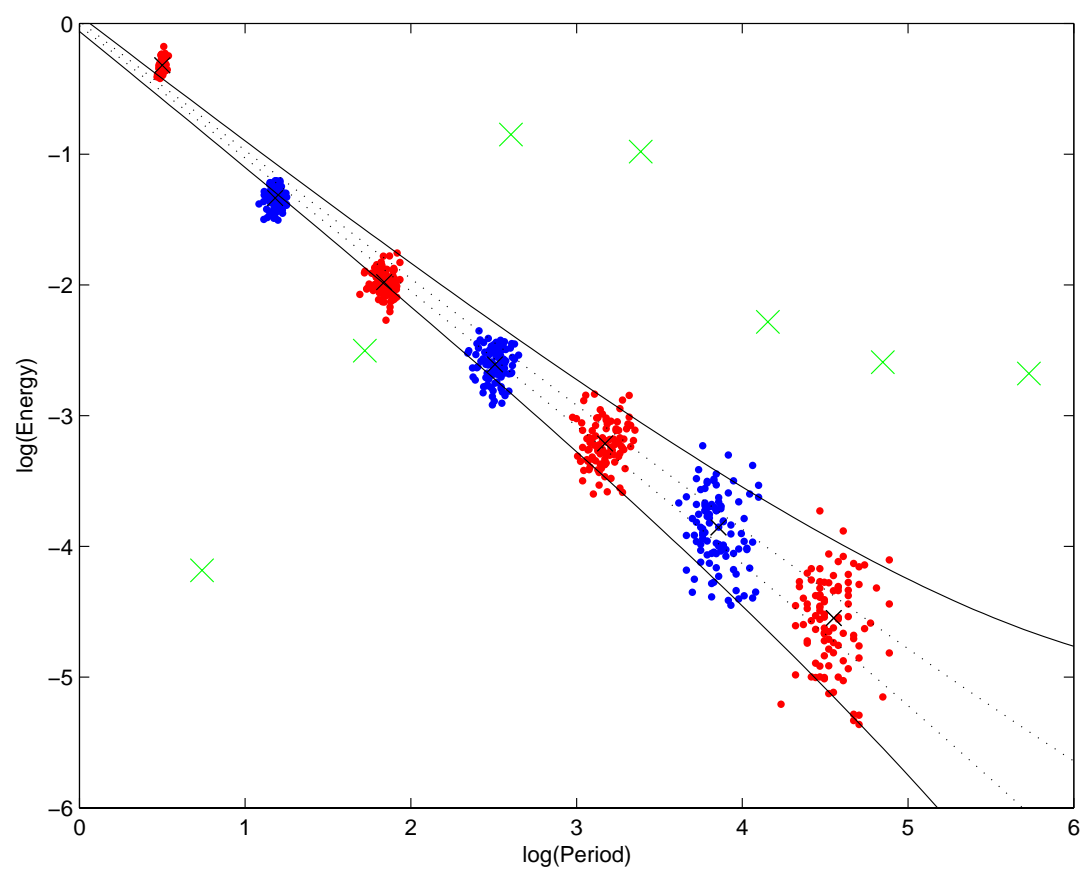

Fig. 3. Log-log diagram of the energy and dominant period of the different intrinsic modes identified by the EMD technique. The separation between observations (green crosses) and Monte-Carlo simulations of white-noise (red and blue dots) displayed in this figure indicate that a range of atmospheric waves can be observed in the Scott Base MF radar data. The black crosses in this figure indicate the center of mass for the individual IMFs produced by the Monte-Carlo simulations. The dotted and full lines indicate the theoretical bounds $(75 \%$ and $99 \%$ limits) within which white-noise should fall.

Monte-Carlo modes observed in Fig. 3. The fact that the dominant periods of the IMFs associated with the MF radar data are less evenly spaced is therefore also a positive indication that this data contains signals at a range of frequencies. In particular, the fact that the first three modes, associated with gravity waves, are offset is suggestive that these IMFs contain signals.

Coughlin and Tung (2004) demonstrated that the EMD technique can be used to separate natural variations in the atmosphere from those associated with red-noise (a Fourier spectrum with increasing power for decreasing frequency) in a similar manner. It is necessary with their technique to assume that the first IMF is purely related to red-noise. This seems to be a reasonable assumption in this case and even if the second IMF was utilized the results do not change. An analysis to calculate the first-order autoregressive model associated with the first IMF is then performed. These autoregressive properties are then used to produce a set of MonteCarlo simulations for red-noise which are compared to the observations in a similar manner to that described in $\mathrm{Wu}$ and Huang $(2004,2005)$. Figure 4 displays the Monte-Carlo simulations and the radar observation IMFs. The full lines in Fig. 4 again represent the range of values within which 75 and 99 percent of the simulations produced using purely white-noise would occur and are displayed for comparison purposes. We note that the Monte-Carlo simulations for red- noise processes lie marginally above the theoretical lines for white-noise processes as would be expected because a rednoise process should have more energy at increasing periods compared to those associated with a white-noise process. However, the values associated with the IMFs, other than the first, produced by analyzing the MF radar data are still well separated from the Monte-Carlo simulations; this suggests that the data is also significantly different from a purely rednoise process. In addition, given that the dominant period associated with the second IMF is of the order of $2 \mathrm{~h}$ it can be proven that the MF radar measurements can observe internal gravity waves. The application of the techniques described in Wu and Huang $(2004,2005)$ and Coughlin and Tung (2004) to the MF radar zonal wind time series suggests that the Scott Base data is sufficiently sensitive to observe internal gravity waves, tides and planetary waves using the EMD technique.

\subsection{Potential for identifying non-linear interactions}

Theoretical studies, such as Teitelbaum and Vial (1991), have proposed mechanisms which can explain the non-linear interactions between tides and planetary-scale waves and also indicated how these interactions might contribute to the variability of atmospheric tides. This section describes an initial study of the potential of the EMD time series analysis technique and the Hilbert-Huang spectrum in studying non-linear 


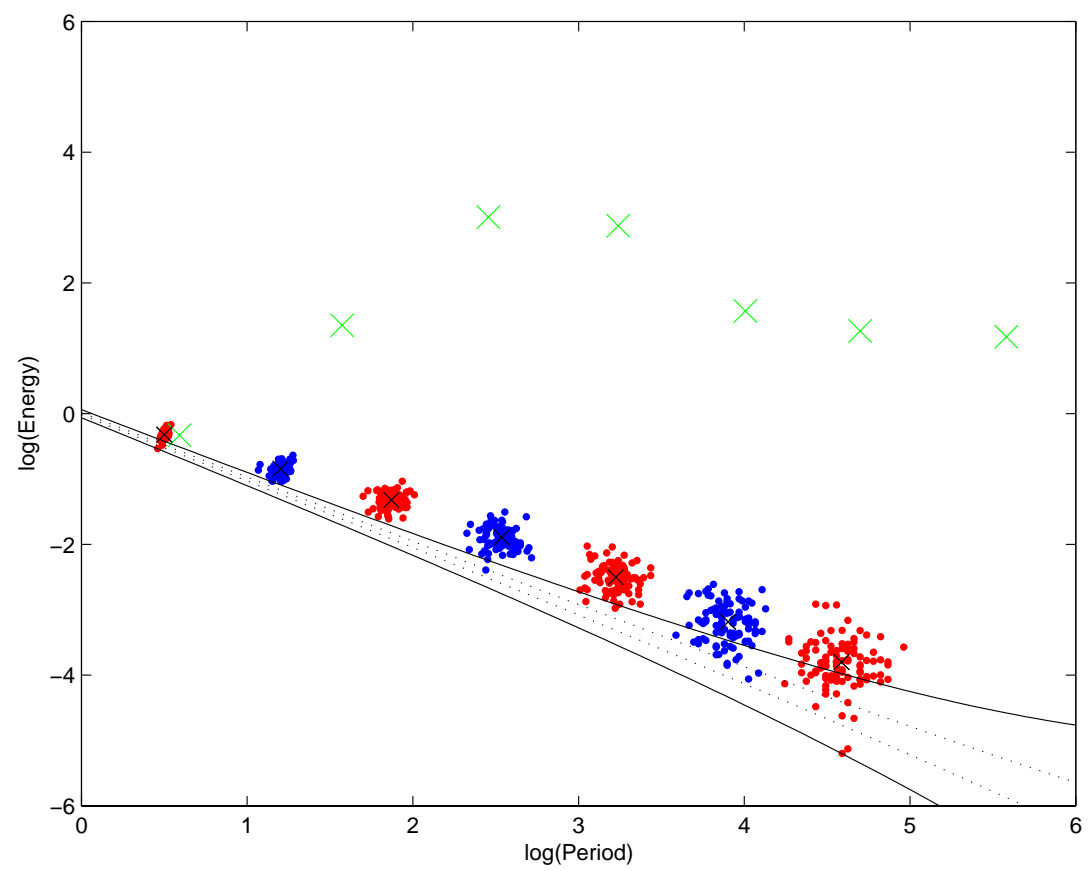

Fig. 4. Log-log diagram of the energy and dominant period of the different intrinsic modes identified by the EMD technique. The separation between observations (green crosses) and Monte-Carlo simulations of red-noise (red and blue dots) displayed in this figure indicate that a range of atmospheric waves can be observed in the Scott Base MF radar data. The black crosses in this figure indicate the center of mass for the individual IMFs produced by the Monte-Carlo simulations. The dotted and full lines indicate the theoretical bounds $(75 \%$ and $99 \%$ limits) within which white-noise should fall.
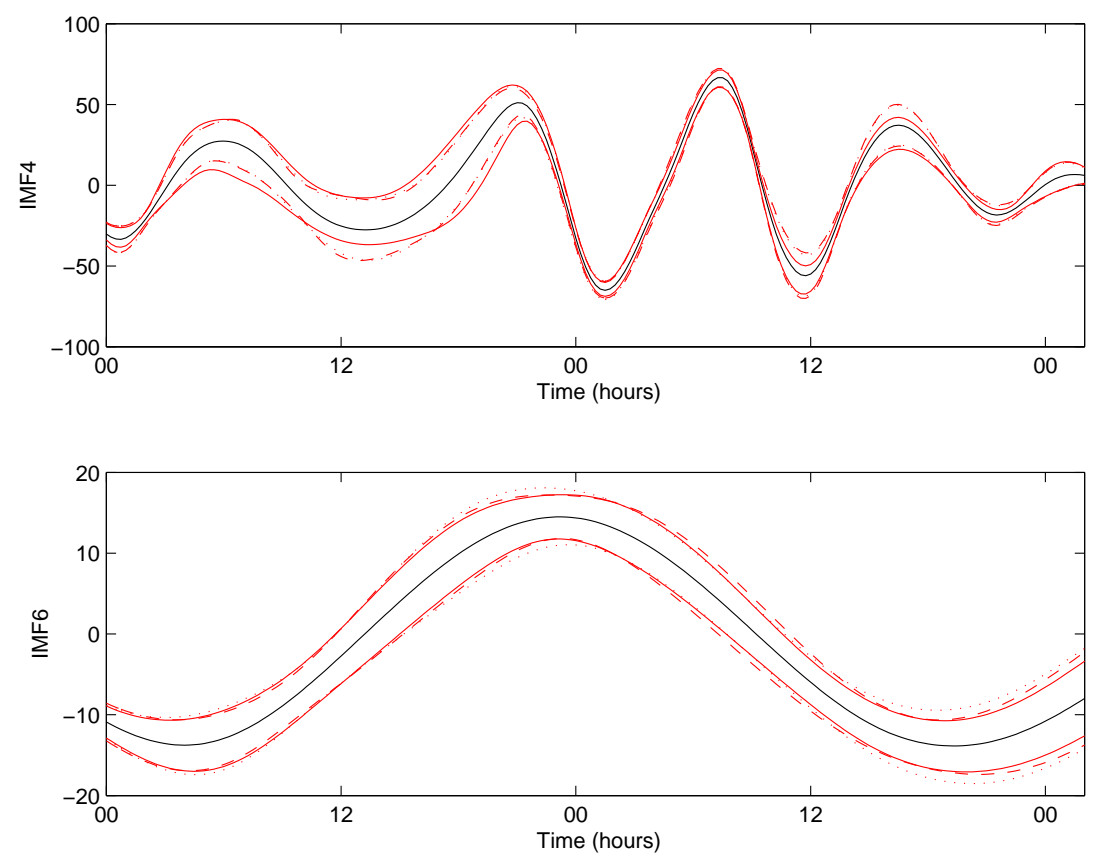

Fig. 5. The ensemble mean (black line) and the mean plus and minus the ensemble standard deviation for realizations which add 1,2 and $5 \%$ white-noise (red full, dotted and dashed lines) for the fourth IMF (a) and the sixth IMF (b) which result from the application of the EMD technique to the zonal wind time-series observed with the Scott Base MF radar at $90 \mathrm{~km}$ between 10 and 12 January 2006 inclusive. 

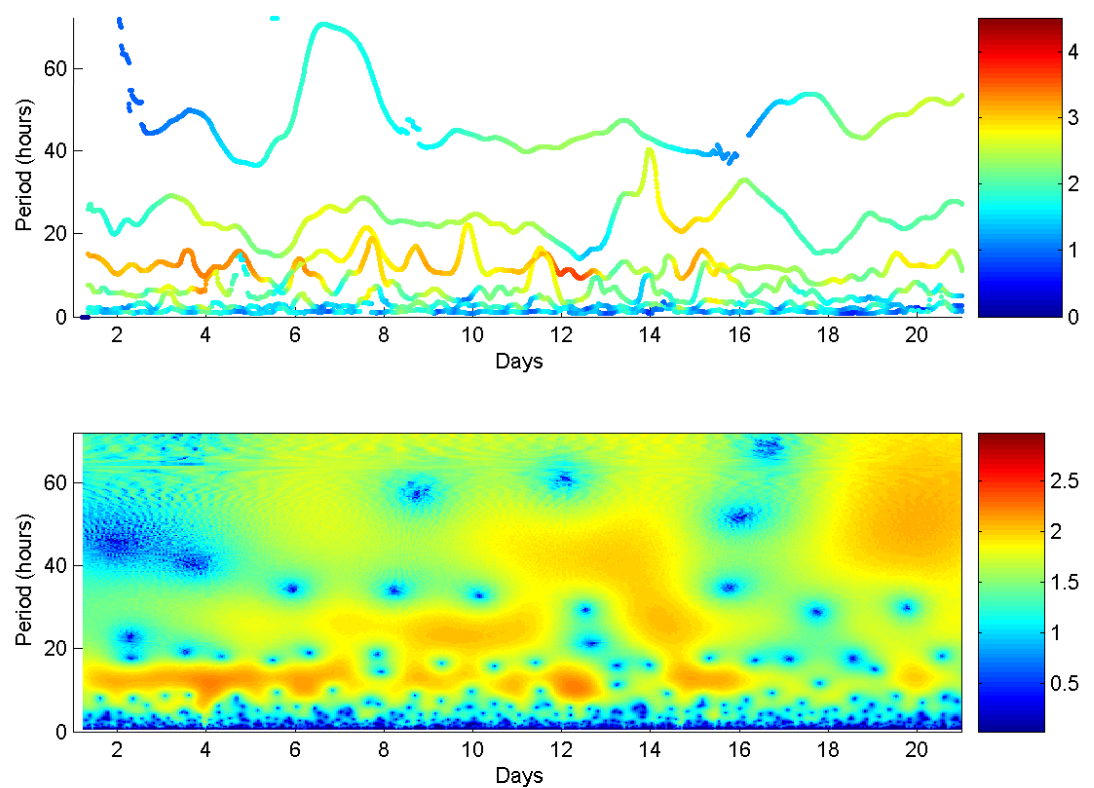

Fig. 6. A skeleton diagram of the Hilbert-Huang spectrum (a) and a contoured diagram of the continuous wavelet transform (b) applied to the time series of zonal winds observed at $90 \mathrm{~km}$ by the Scott Base MF radar over the period 1 to 20 January 2006.

wave-wave interactions. Figures $5 \mathrm{a}$ and $\mathrm{b}$ display the ensemble means, for the 500 realizations of the data series, and the standard deviations of the ensemble when 1,2 and $5 \%$ noise is added for the fourth and sixth IMFs, respectively, for the period between 10 and 12 January 2006 inclusive. The fact that the lines representing the standard deviations associated with the ensembles are so similar indicates that these values provide an accurate measure of the uncertainty associated with specific features in IMFs and that these uncertianties are relatively unaffected by the choice of the magnitude of the white-noise added. The fourth IMF which has a dominant period of $12 \mathrm{~h}$ and is therefore related to the semi-diurnal tide displays a clear amplitude modulation with a period similar to two days. The maximum peak-peak semi-diurnal tidal amplitudes are observed between midnight and noon on the 11 January 2006. Comparison of the approximately twoday modulation of the semi-diurnal tide with the sixth IMF, which is likely to be associated with the quasi-two-day planetary wave, displays a clear similarity suggesting that the modulation of the semi-diurnal tide may be related to the quasi-two-day wave. It should be noted that the period of the quasi-two-day wave is close to $42 \mathrm{~h}$ in this case. It is possible that this relatively short period for the quasi-two-day wave could be associated with mode-mixing which was discussed previously in Sect. 2. However, this value for the period of the quasi-two-day wave does fall within the range of periods previously identified as related to the quasi-two-day wave (Pancheva, 2006). The modulation of the semi-diurnal tide with a period similar to two days is highly indicative of a nonlinear wave-wave interaction between these modes (Pancheva et al., 2000, 2002; Pancheva and Mitchell, 2004; Pancheva, 2006).
Teitelbaum and Vial (1991) indicates that a non-linear interaction in the advection terms of the momentum and thermodynamic equations occurs between two "primary waves". The interaction generates a family of "secondary waves", including one wave with a frequency, wave number and phase which is the sum of the frequencies, wave number and phase of the primary waves, and hence is called the "sum wave"; and another wave whose parameters are the difference of these values, and so is called the "difference wave". These secondary waves may then beat with the primary waves and modulate the amplitude of the shorter period primary wave at the period of the longer period primary wave. In the case of such a non-linear coupling between primary waves that are a planetary wave and a tide, the result is a periodic modulation of the amplitude of the tide at the period of the planetary wave. In addition the theory discussed in Teitelbaum and Vial (1991) suggests that an observation which includes the two primary waves associated with a nonlinear interaction can only occur in the region where the interaction takes place. Thus, this suggests that a localized nonlinear interaction between the two-day wave and semi-diurnal wave may be observed in this case. Simple Fourier analysis around this region displays peaks close to the beat frequencies between the two primary waves (the sum and difference of the frequencies of the two primary waves) which Teitelbaum and Vial (1991) identify as a necessary condition for this type of nonlinear interaction to be identified (not shown). However, Riggin et al. (2003) indicated that refractive effects may also be relevant to the modulation of semi-diurnal tidal amplitudes at planetary wave periods and thus it is not possible to unambiguously identify this event as a non-linear wavewave interaction. 
To examine the amplitude modulation identified in Fig. 5 in an alternative manner, Fig. 6a displays a skeleton diagram of the Hilbert-Huang spectrum (see Sect. 2 and references in Huang and Shen, 2005, for details). It should be noted that the skeleton diagram is generally the most desirable form of presentation because in this manner the instantaneous period and energy variations associated with individual IMFs as a function of time can generally be identified. The color of the lines in Fig. 6a indicate the amplitude squared of the mode as a function of time. The form of the line represents the variation of the instantaneous period of the IMF as a function of time (see Huang and Shen, 2005, for details). Figure $6 \mathrm{~b}$ displays the continuous wavelet transform for the same dataset and is shown to aid in the interpretation of Fig. 6a. The continuous wavelet analysis presented uses the Morlet wavelet as the "mother wavelet", this consists of a plane wave modulated by a Gaussian envelope. The Morlet wavelet was selected because of its simplicity and resemblance to the modulated wave "packets" and its previous use in studies which have examined non-linear interactions between waves (see Pancheva et al., 2002; Pancheva and Mitchell, 2004; Pancheva, 2006). However, it should be borne in mind that the choice of the wavelet type imposes biases in the analysis of the wave bursts and therefore the magnitude of the wavelet coefficient displayed while being related to the amplitude of the oscillation only provides a qualitative measure. Comparison of Fig. 6a with Fig. 6b suggests that the amplitudes obtained by the two methods at specific points in the time-period parameter space have a similar form. However, examination of the color scales in Fig. 6 shows that the color scale associated with the Continuous wavelet transform has been selected for comparison purposes only and does not represent a quantitative measure of the amplitudes of the motions unlike the Hilbert-Huang spectrum. Comparison of Figs. 6a and $\mathrm{b}$ also indicates that the HilbertHuang spectrum shows finer time resolution than observed in the continuous wavelet transform representation where the time resolution at longer periods is particularly coarse. Each colored line in the skeleton diagram (Fig. 6a) relates to an individual IMF and therefore examination of an individual line can give us an idea of the amount of amplitude and frequency modulation on a particular mode. Thus, this representation seems to have great potential for the examination of nonlinear interactions. For example, examination of Fig. 6a shows a strong amplitude modulation close to $12 \mathrm{~h}$ periods around the 11 January 2006 which was also displayed in Fig. 5. This region of amplitude modulation in the semi-diurnal tide corresponds to a region of strong enhancement of the amplitude of the quasi-two-day wave, the actual instantaneous period in this region being close to $44 \mathrm{~h}$ (see Fig. 6a). Given that nonlinear interactions are most likely to occur between waves with large amplitudes this is therefore a feature in the data which might be expected. Another advantage of this technique relative to the continuous wavelet transform is that it only displays information where it is available rather than a smoothed representation. Thus, the likelihood of errors in interpretation are reduced. Further examination of the potential of the Hilbert-Huang spectrum for the analysis of non-linear interactions will be the subject of future work.

\section{Conclusions}

This study suggests that careful application of the Empirical Mode Decomposition (EMD) technique can decompose a time-series into physically meaningful modes. In this case, the dominant IMFs obtained from the MF radar data are associated with a range of atmospheric waves, namely gravity waves, tides and planetary waves. Application of the techniques described in Wu and Huang $(2004,2005)$ and Coughlin and Tung (2004) allows us to show that the output when the EMD technique is applied to the MF radar data is significantly different from that which could result from whiteand red-noise processes, respectively. The combination of the newly developed EMD algorithm with the improved MF radar measurements means that we are able to observe gravity waves for the first time.

Utilization of the EMD technique also allows us to observe an amplitude modulation of the semi-diurnal tide which seems to be associated with the presence of the two-day wave. This demonstrates that the technique allows us to observe non-linear interactions between waves. Examination of the Hilbert-Huang spectrum also allows the wave-field to be observed in an alternative form, similar to that resultant from the continuous wavelet transform technique. However, the fact that the basis function of EMD is data-driven and does not need to be selected a priori is a major advantage. In addition, the skeleton diagram provides a method of presentation which allows quantitative information on the instantaneous period and amplitude squared to be displayed as a function of time. Thus, it provides a novel way to view frequency and amplitude-modulated wave phenomena and potentially non-linear interactions. Future work will provide a more detailed analysis of the potential of the EMD technique and the Hilbert-Huang spectrum to examine non-linear wave-wave interactions.

Acknowledgements. We would like to thank P. Flandrin for making his matlab implementation of the EMD algorithm freely available on the web (http://perso.ens-lyon.fr/patrick.flandrin/emd.html). Logistic support for the Scott Base radar is provided by Antarctica New Zealand. We acknowledge the work by the Scott Base and University of Canterbury technical staff to ensure continuous operation. Financial support for this project has been provided by the University of Canterbury via grant U6561 to A. J. McDonald. We would like to thank two anonymous reviewers for helpful comments which have improved this manuscript.

Topical Editor U.-P. Hoppe thanks two referees for their help in evaluating this paper. 


\section{References}

Baumgaertner, A. J. G., McDonald, A. J., Fraser, G. J., and Plank, G. E.: Long-term observations of mean winds and tides in the upper mesosphere and lower thermosphere above Scott Base, Antarctica, J. Atmos. Solar-Terr. Phys., 67, 1480-1496, 2005.

Baumgaertner, A. J. G., Jarvis, M. J., McDonald, A. J., and Fraser, G. J.: Observations of the wavenumber 1 and 2 components of the semi-diurnal tide overAntarctica, J. Atmos. Solar-Terr. Phys., 68, 1195-1214, 2006.

Coughlin, K. and Tung, K. K.: Eleven-year solar cycle signal throughout the lower atmosphere, J. Geophys. Res.-Atmos., 109, D21105, doi:10.1029/2004JD004873, 2004.

Huang, N. E. and Shen, S. S.: The Hilbert-Huang transform and its applications, Interdisciplinary mathematical sciences; v. 5, World Scientific, New Jersey, 2005.

Huang, N. E., Shen, Z., Long, S. R., Wu, M. L. C., Shih, H. H., Zheng, Q. N., Yen, N. C., Tung, C. C., and Liu, H. H.: The empirical mode decomposition and the Hilbert spectrum for nonlinear and non-stationary time series analysis, Proceedings of the Royal Society of London Series a-Mathematical Physical and Engineering Sciences, 454, 903-995, 1998.

Huang, N. E., Wu, M. L. C., Long, S. R., Shen, S. S. P., Qu, W. D., Gloersen, P., and Fan, K. L.: A confidence limit for the empirical mode decomposition and Hilbert spectral analysis, Proceedings of the Royal Society of London Series a-Mathematical Physical and Engineering Sciences, 459, 2317-2345, 2003.

Janosi, I. M. and Muller, R.: Empirical mode decomposition and correlation properties of long daily ozone records, Phys. Rev. E, 71, 056 126, 2005.

Kalnay, E., Kanamitsu, M., Kistler, R., Collins, W., Deaven, D., Gandin, L., Iredell, M., Saha, S., White, G., Woollen, J., Zhu, Y., Chelliah, M., Ebisuzaki, W., Higgins, W., Janowiak, J., Mo, K. C., Ropelewski, C., Wang, J., Leetmaa, A., Reynolds, R., Jenne, R., and Joseph, D.: The NCEP/NCAR 40-year reanalysis project, Bull. Amer. Meteorol. Soc., 77, 437-471, 1996.

Malinga, S. B. and Poole, L. M. G.: The 16-day variation in the mean flow at Grahamstown (33.3 degrees S, 26.5 degrees E), Ann. Geophys., 20, 2027-2031, 2002a.

Malinga, S. B. and Poole, L. M. G.: The 16-day variation in tidal amplitudes at Grahamstown (33.3 degrees S, 26.5 degrees E), Ann. Geophys., 20, 2033-2038, 2002 b.

Pancheva, D., Beard, A., Mitchell, N. J., and Muller, H. G.: Nonlinear interactions between planetary waves in the mesosphere/ lower thermosphere region, J. Geophys. Res., 105, 157-170, 2000 .
Pancheva, D., Merzlyakov, E., Mitchell, N. J., Portnyagin, Y., Manson, A. H., Jacobi, C., Meek, C. E., Luo, Y., Clark, R. R., Hocking, W. K., MacDougall, J., Muller, H. G., Kurschner, D., Jones, G. O. L., Vincent, R. A., Reid, I. M., Singer, W., Igarashi, K., Fraser, G. I., Fahrutdinova, A. N., Stepanov, A. M., Poole, L. M. G., Malinga, S. B., Kashcheyev, B. L., and Oleynikov, A. N.: Global-scale tidal variability during the PSMOS campaign of June-August 1999: interaction with planetary waves, J. Atmos. Solar-Terr. Phys., 64, 1865-1896, 2002.

Pancheva, D. V.: Quasi-2-day wave and tidal variability observed over Ascension Island during January/February 2003, J. Atmos. Solar-Terr. Phys., 68, 390-407, 2006.

Pancheva, D. V. and Mitchell, N. J.: Planetary waves and variability of the semidiurnal tide in the mesosphere and lower thermosphere over Esrange (68 degrees N, 21 degrees E) during winter, J. Geophys. Res.-Space Physics, 109, A08307, doi:10.1029/2004JA010433, 2004.

Riggin, D. M., Meyer, C. K., Fritts, D. C., Jarvis, M. J., Murayama, Y., Singer, W., Vincent, R. A., and Murphy, D. J.: MF radar observations of seasonal variability of semidiurnal motions in the mesosphere at high northern and southern latitudes., J. Atmos. Solar Terr. Phys., 65, 483-493, 2003.

Rilling, G., Flandarin, P., and Gonclaves, P.: On Empirical Mode Decomposition and its algorithms, in IEEE-EURASIP Workshop on Nonlinear Signal and Image Processing - NSIP-03, Grado, Italy, 2003.

Teitelbaum, H. and Vial, F.: On Tidal Variability Induced by Nonlinear-Interaction with Planetary-Waves, J. Geophys. Res.Space Physics, 96, 14 169-14 178, 1991.

Torrence, C. and Compo, G. P.: A practical guide to wavelet analysis, Bull. Amer. Meteorol. Soc., 79, 61-78, 1998.

Wu, Z. H. and Huang, N.: Statistical Significance Test of Intrinsic Mode Functions, in: Hilbert-Huang Transform and its application, edited by: Huang, N. E. and Shen, S. S. P., pp. 107-127, World Scientific, 2005.

Wu, Z. H. and Huang, N. E.: A study of the characteristics of white noise using the empirical mode decomposition method, Proceedings of the Royal Society of London Series a-Mathematical Physical and Engineering Sciences, 460, 1597-1611, 2004.

Zhu, X., Shen, Z., Eckermann, S. D., Bittner, M., Hirota, I., and Yee, J. H.: Gravity wave characteristics in the middle atmosphere derived from the Empirical Mode Decomposition method, J. Geophys. Res.-Atmos., 102, 16 545-16 561, 1997. 